\title{
A NEW TYPE OF NON-LINEAR APPROXIMATION WITH APPLICATION TO THE DUFFING EQUATION
}

\author{
Chao Lin Lou' and David L. Sikarskie \\ Department of Aerospace Engineering. The University of Michigan. Michigan. U.S.A.

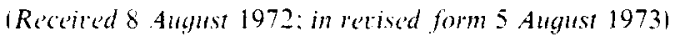

\begin{abstract}
A new type of trial solution which differs from the usual linear combination of approximating functions is considered. It involves modifying the approximating functions with "form functions:" functions containing undetermined parameters appearing non-linearly, the proper choice of which provide a closer approximation to the large local curvatures which appear in some non-linear problems. In this paper the "form function" approximation is demonstrated for steady-state solutions of the Duffing equation. This equation arises in the problem of non-linear vibration of buckled beams and plates. It is shown that the stability behavior of these steady-state solutions is governed by a Hill equation. It is found that the "form function" approximation gives noticeably better numerical results than. for example. those given by the harmonic balance method. The method also provides additional insight into the non-linear behavior. particularly in the low frequency response region.
\end{abstract}

\section{INTRODUCTION}

In applying the method of weighted residuals.[1] e.g.. Galerkin's method, method of least squares. etc.. to non-linear structural mechanics problems. one must first assume a "trial" solution. Generally this trial solution is a linedr combination of a set of approximating functions (multiplied by unknown coefficients). all of which satisfy the necessary constraints in the problem. Such a linear combination has the strong advantages of simplicity and existing formalism. [1] In certain problems. however. it can be inadequate numerically. One such example is a class of problems which exhibit large local curvatures in the dependent variable or variables. ${ }_{*}^{*}$ Solutions in these large curvature regions using a linear combination of approximating functions are often either poor (particularly derivatives of the solution, e.g.. stresses) or require adding many terms. usually requiring significant effort. There appears to be no systematic study in the literature of other types or combinations of approximating functions which might be useful for such problems.

In the present paper a type of trial solution is proposed which is both efficient in such situations (at least for the problems investigated to date) and useful in terms of gaining additional insight into the non-linear behavior. It involves modifying the usual trial solution with "form functions:" functions containing unknown parameters appearing non-linearly the proper choice of which approximate the desired local effects. Details are included in the following section.

The particular problem for which the method will be applied is the forced. steady state response of the following form of Duffing equation;

$$
\frac{\mathrm{d}^{2} \zeta}{\mathrm{d} \tau^{2}}+p(1-\lambda) \xi q \check{\zeta}^{3}=F(\tau)
$$

\footnotetext{
$\uparrow$ Presently with Light Truck Design Analysis. Ford Motor Company. Dearborn. Michigan. U.S.A.

†Consider specifically. say. beams plates having concentrated loadings and vanishing flexural rigidity.
} 
where $F(\tau)$ is periodic. An equation of this type arises in the non-linear vibration of beams/plates subjected to axial/membrane loading, see [2,3,4]. In the base of beams for the value $\lambda=1$, the axial load is the static buckling load while for $\lambda=0$, the axial load vanishes and for $\lambda>1$ the beam is vibrating in a post buckled state.

Note that two motions are physically possible in the post buckled case, a one-sided vibration about the buckled configuration and a dynamic snap-through. In its present form equation (1) pertains to the dynamic snap-through response.

The purpose of the present paper is to provide an alternative approach to the methods currently used for obtaining periodic solutions to the above equation, see $[5,6,7]$, which is particularly useful both numerically and for gaining additional insight into the non-linear behavior. It is also pointed out later that this approach is useful for other similar non-linear problems.

\section{II. "FORM FUNCTION" APPROXIMATION}

Exact solutions to equation (1) for a general forcing function do not exist. Exact solutions are available in terms of elliptic functions, however, for both the homogeneous equation. Burgreen, [8] and for forcing functions of the elliptic type. Iwan[9] uses this latter result in an "equivalent equation" approach, i.e.. an equivalent (auxiliary) differential equation for which a known exact periodic solution exists is defined and the difference between the two equations minimized. A more direct approach is considered here. We look for an approximate solution of the form

$$
\xi(\tau)=\sum_{i=1}^{n} G_{i}\left(\tau ; k_{i 1}, \ldots, k_{i j}\right) \check{\zeta}_{i}(\tau)
$$

where $\xi_{i}(\tau)$ are terms of the usual periodic approximation, generally trigonometric. The $G_{i}$ 's can be thought of physically as "form functions," which are capable of reffecting local effects (e.g., large curvatures) in the approximation by proper adjustment of the as yet unknown parameters $k_{i j}$. In effect, the functions $G_{i}$ improve the convergence of the series. Two questions arise. How are the functions $G_{i}$ generated and once found do they represent an efficient approximation in the various approximation schemes. The first question is the more difficult from a theoretical viewpoint and is only partially answered in the present paper, while the second (and major thrust of the paper) is dealt with in detail in the following sections.

To demonstrate the generation of the $G_{i}$ 's. we consider the first term of the series (2) with $G_{1}$ dependent on a single parameter $k$. Thusं

$$
\xi(\tau, k)=G(\tau ; k) \xi(\tau)
$$

We further consider $F(\tau)=B \cos \omega \tau$. where $B$. () are given. For this forcing function we chose $\xi(\tau)=A \cos \omega \tau ; A$ is an unknown amplitude. This is the usual lead term in the harmonic balance method. If equation (3) is substituted into equation (1) the following residual $R$ results

$$
R=\left(\ddot{G}-\omega^{2} G+p(1-\dot{\lambda}) G-\frac{B}{A}\right) A \cos (\tau)-2 \omega A \dot{G} \sin \omega \tau+q A^{3} G^{3} \cos ^{3} \omega \tau, \dot{\mathrm{d}} \equiv \frac{\mathrm{d}}{\mathrm{d} \tau} .
$$

The following integral, which is a measure of the error. is defined,

$$
J=\int_{0}^{2 \pi ; \omega} R^{2} \mathrm{~d} \tau=\int_{0}^{2 \pi i \omega} F(\tau, G, \dot{G} \cdot \ddot{G}) \mathrm{d} \tau
$$

+ Subscripts are dropped to simplify notation. 
A necessary condition for $J$ to be a minimum is that it satisfies the Euler equation, i.e.,

$$
\frac{\partial F}{\hat{C} G}-\frac{\mathrm{d}}{\mathrm{d} \tau}\left(\frac{\partial F}{\partial G}\right)+\frac{\mathrm{d}^{2}}{\mathrm{~d} \tau^{2}}\left(\frac{\hat{\partial} F}{\hat{c} G}\right)=0 .
$$

Substituting equations (4) and (5) into equation (6) the following non-linear differential equation in $G$ results

$$
\begin{aligned}
& \dddot{G} \cos \left(\omega \tau-4 \omega \dddot{G} \sin \omega \tau+2\left[-3 \omega^{2}+p(1-\lambda)\right] G \cos \omega \tau\right. \\
& +4 \omega\left[\omega^{2}-p(1-\lambda)\right] \dot{G} \sin \omega \tau+\left[\omega^{2}-p(1-\lambda)\right]^{2} G \cos \omega \tau+\frac{B}{A}\left[\omega^{2}-p(1-\lambda)\right] \cos \omega \tau \\
& +3 q\left\{\left[2 \omega^{2} \cos \omega \tau \sin ^{2} \omega \tau-2 \omega^{2} \cos ^{3}\left(\omega \tau+\frac{4}{3} p(1-\lambda) \cos ^{3} \omega \tau\right] G^{3}\right.\right. \\
& -8 \omega \cos ^{2} \omega \tau \sin \omega \tau G^{2} G+2 \cos ^{3} \omega \tau G G^{2}+2 \cos ^{3} \omega \tau G^{2} \dot{G} \\
& \left.-\frac{B}{A} \cos ^{3} \omega \tau G^{2}+q A^{2} \cos ^{5} \omega \tau G^{5}\right\} A^{2}=0 .
\end{aligned}
$$

Boundary conditions for $G$ are

$$
\begin{array}{lll}
G(\tau, k)=1 & \text { at } & \tau=0 . \pi / \omega \\
G(\tau, k)=0 & \text { at } & \tau=0 . \pi / \omega
\end{array}
$$

In addition to periodicity of $G$. we expect (for the snap-through vibration) symmetry about $\tau=0, \pi / 2 \omega, \pi / \omega, 3 \pi / 2 \omega, 2 \pi / \omega, \ldots$.

A closed form, analytic solution for $G$ is not available. Instead, we look for a perturbation solution $(q \ll 1)$ of the form

with

$$
G(\tau, k)=1+k f(\tau)+k^{2} g(\tau)+\ldots
$$

$$
k=a_{1} q+a_{2} q^{2}+\ldots
$$

and $f(\tau), g(\tau), \ldots$ are functions to be determined. If the solution is expanded about the linear, natural frequency $\sqrt{ } p(1-\lambda) . \dagger$ and further if $B$ is assumed small,.then

$$
\begin{gathered}
\omega^{2}-p(1-\lambda)=b_{1} q+b_{2} q^{2}+\ldots \\
\frac{B}{A}=c_{1} q+c_{2} q^{2}+\ldots
\end{gathered}
$$

If equations (9)-(12) are substituted into equations (7), (8) and the results ordered in powers of $g$, the following boundary value problems in $f(\tau) . g(\tau)$ result (carrying the solution through two terms):

$$
\begin{aligned}
& a_{1}\left\{-\dddot{f} \cos \omega \tau+4 \omega \dddot{f} \sin \omega \tau+4 \omega^{2} \ddot{f} \cos \omega \tau\right\}-2 \omega^{2} A^{2}\left\{3 \cos \omega \tau \sin ^{2} \omega \tau-\cos ^{3} \omega \tau\right\}=0 \\
& a_{1}^{2}\left\{\dddot{g} \cos \omega \tau-4 \omega \dddot{g} \sin \omega \tau-4 \omega^{2} \ddot{g} \cos \omega \tau\right\} \\
& +a_{2} \dddot{f} \cos \omega \tau-4 a_{2} \omega \dddot{f} \sin \omega \tau-\left\{4 a_{2} \omega \omega^{2}+2 a_{1} b_{1}-6 a_{1} A^{2} \cos ^{2} \omega \tau\right\} \ddot{f} \cos \omega \tau \\
& +\left\{4 a_{1} b_{1} \omega-24 a_{1} \omega \cos ^{2} \omega \tau\right\} \int \sin \omega \tau+\left\{18 a_{1} \omega^{2} \sin ^{2} \omega \tau-6 a_{1} \omega^{2} \cos ^{2} \omega \tau\right\} A^{2} f \cos \omega \\
& \quad+\left\{b_{1}^{2}+b_{1} c_{1}\right\} \cos \omega \tau-\left\{4 b_{1} A+3 c_{1}\right\} A \cos ^{3} \omega \tau+3 A^{4} \cos ^{5} \omega \tau=0
\end{aligned}
$$


with

$$
\begin{array}{ll}
f(\tau)=0, & g(\tau)=0 \\
\dot{f}(\tau)=0, & \dot{g}(\tau)=0
\end{array} \quad \text { at } \quad \tau=0 . \pi(1)
$$

The solution to equations (13). (15) can be shown to be

$$
\begin{gathered}
f(\tau)=-\sin ^{2} \omega \tau \\
a_{1}=\frac{A^{2}}{8()^{2}}
\end{gathered}
$$

With $f(\tau), a_{1}$ known, equations (14), (15) can be solved to give

$$
g(\tau)=\sin ^{4}(\omega \tau
$$

with $a_{2}, b_{1}, c_{1}$ determined from the following two coupled. non-linear algebraic equations

$$
\begin{aligned}
48 a_{2}()^{4}+\frac{3}{2} h_{1} A^{2}-b_{1}^{2}-b_{1} c_{1} & =\frac{9}{8} A^{+} \\
64 a_{2}()^{4}-2 b_{1} A^{2}-3 c_{1} A & =-\frac{3}{4} A^{+}
\end{aligned}
$$

For a given forcing function. i.e., fixed $c_{1}$, equations (18) admit two sets of solutions for $a_{2}, b_{1}$ while $a_{1}$ is determined uniquely from equation (16). The form of $G(\tau, k)$ is also determined uniquely

$$
G(\tau, k)=1-k \sin ^{2} \omega \tau+k^{2} \sin ^{4} \omega \tau+\ldots
$$

As discussed previously. what is of interest in the above arguments is not the perturbation solution per se, but the form of $G(\tau, k)$. equation (19). $G(\tau . k)$ will be used directly in equation (3) with $k$ an unknown parameter to be determined by whatever approximation scheme is chosen. Since the full non-linear range is of interest. $G(\tau, k)$ in the form of equation (19) will be replaced by a function with the same asymptotic behavior. Functions which suggest themselves are $\left[1+k \sin ^{2}(1) \tau\right]^{-1}$ and for a limited range $e^{-k \sin ^{2}(i)}$. The choice of function is now governed by its behavior for large non-linearities and such practical questions as ease of integrability in the approximating scheme. etc. For these reasons. $\mathrm{e}^{-k \sin ^{2} \cdots \tau}$ will be used. Additional work is underway using asymptotic methods to establish further criteria for selecting the $G_{i}$ s.

The form function $G(\tau, k)=\mathrm{e}^{-k \sin ^{2} \omega \tau}$ has several interesting features. It has the capability of introducing large curvatures at $(j \tau=0, \pi .2 \pi \ldots$ shown by the Jacobian elliptic functions in the exact solution (free vibration case). see [8]. Large local curvatures can also be viewed as the appearance of higher harmonics. Note that although the approximation. equation (3). is a two parameter approximation it contains the higher harmonics and thus should provide better accuracy than the usual two term harmonic batance (for comparable numerical effort). Although $G(\tau, k)$ is established formally only for $q \ll 1$. the numerical results obtained indicate a much wider range of validity. The only exception to this is the low frequency forced response where the higher harmonics enter strongly.[10] Here the approximation (3) yields good results in an averaged sense. i.e.. a reasonably accurate amplitude frequency plot. but it does not capture all of the response details. Additional terms of series (2) are needed here. 


\section{RESPONSE}

Consider equation (1) with $F(\tau)=B \cos (\omega \tau$. We seek an approximate periodic solution of the form.

$$
\boldsymbol{\xi}(\tau)=A \mathrm{e}^{-k \sin ^{2}(\omega \tau} \cos \omega \tau
$$

If equation (20) is substituted into equation (1), the following residual results

$$
R(\xi)=\frac{\mathrm{d}^{2} \xi}{\mathrm{d} \tau^{2}}+p(1-\lambda) \xi+q \xi^{3}-B \cos (\omega \tau .
$$

To minimize $R(\xi)$ with respect to $A . k$. the method of weighted residuals is used

$$
\begin{gathered}
\int_{0}^{2 \pi / \omega} R(\xi) \xi \mathrm{d} \tau=0 \\
\int_{0}^{2 \pi / \omega} R(\xi) \frac{\partial R}{\partial k} \mathrm{~d} \tau=0 .
\end{gathered}
$$

The weighting functions $\xi . j R / c k$ correspond to Galerkin's method, and the method of least squares respectively. Note that the non-linear appearance of $k$ in equation (20) imposes limitations on the choice of weighting functions. Although alternatives to equations (22), (23) exist, these are used because of the resulting simplicity.

Substituting equation (20) into (21) and then (21) into equations (22). (23) the following coupled transcendental equations in $A . k$ result

$$
\begin{aligned}
A^{3} q[ & \left.z_{0}(2 k)+\left(1-\frac{1}{4 k}\right) z_{1}(2 k)\right] \\
& -A\left\{\left[\omega^{2}(k+1)-p(1-\lambda)\right] z_{0}(k)+\left[\omega^{2}(k-1)-p(1-\lambda)\right] z_{1}(k)\right\} \\
A^{5} q^{2} & {\left[\left(\frac{1}{2 k}-\frac{1}{4 k^{2}}\right) z_{0}(3 k)+\left(\frac{1}{2 k}-\frac{1}{3 k^{2}}+\frac{1}{6 k^{3}}\right) z_{1}\left(\frac{k}{2}\right)+z_{1}\left(\frac{k}{2}\right)\right]=0 } \\
& +A^{3} q\left\{\left[\omega^{2}\left(\frac{3}{2}+\frac{3}{2 k}\right)+p(1-\lambda) \frac{1}{k}\right] z_{0}(2 k)\right. \\
& \left.+\left[\omega^{2}\left(\frac{3}{2}-\frac{3}{2 k}-\frac{3}{2 k^{2}}\right)+p(1-\lambda)\left(\frac{1}{k}-\frac{1}{k^{2}}\right)\right] z_{1}(2 k)\right\} \\
& -A^{2} q B\left[\frac{1}{k} z_{0}\left(\frac{3}{2} k\right)-\left(\frac{1}{k}-\frac{4}{3 k^{2}}\right) z_{1}\left(\frac{3}{2} k\right)\right] \\
& -A\left\{\left[\omega^{4}(12 k+2)+2 \omega^{2} p(1-\lambda)+p^{2}(1-\lambda)^{2} \frac{1}{k}\right] z_{0}(k)\right. \\
& \left.-\left[\omega^{4}\left(3 k+4+\frac{5}{k}\right)+\omega^{2} p(1-\lambda)\left(4+\frac{2}{k}\right)\right] z_{1}(k)\right\}
\end{aligned}
$$

where $z_{0}(x)=\mathrm{e}^{-x} I_{0}(x)$ and $z_{1}(x)=\mathrm{e}^{-x} I_{1}(x) ; I_{0}(x) . I_{1}(x)$ are modified Bessel functions of 
order zero and one respectively. Equation (24) is the counterpart to the usual amplitude frequency equation. Note that as $k \rightarrow 0$. this reduces to the usual one term amplitude frequency equation of [2]. Equation (25) is needed to establish $k$. however. it has no particular physical interpretation. Solution of these equations establishes the response of the system.

The logic used in solving equations (24), (25) is as follows. Equation (24) is a cubic equation in $A$ and has either one or three distinct real roots except for a single point in the frequency spectrum $\left.(\omega)=()^{*}\right)$ where it has two distinct roots (see Fig. 1). (1)* is first located

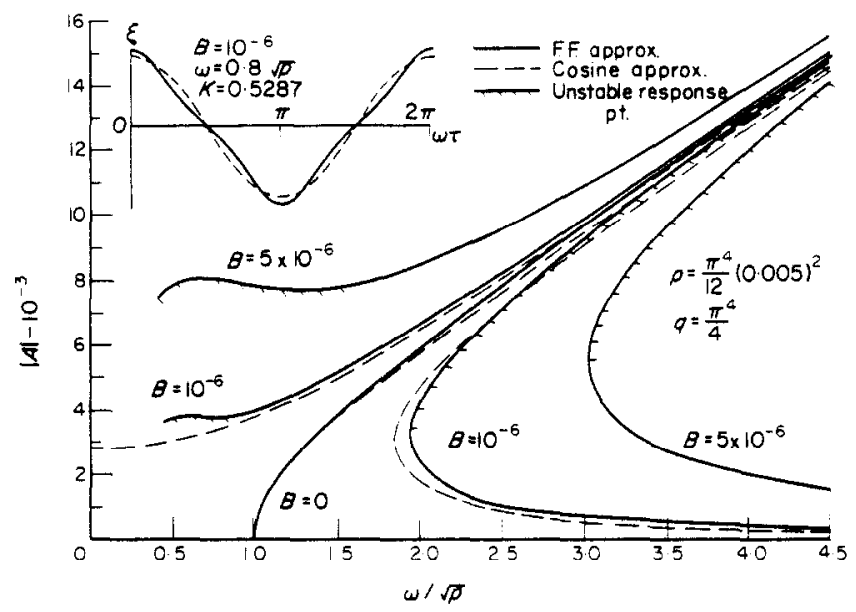

Fig. 1. Frequency-response curves for $i=0$.

using the properties of cubic equations. The Newton-Raphson method[11] is used to find $A, k$ for both regions $\left(\omega<\omega^{*}, \omega>\omega^{*}\right.$. For $\omega>\omega^{*}$ three sets of $A . k$ are obtained. The upper branch is found by extrapolating the $A . k$ solution for $(1)<()^{*}$ and using these values as initial guesses in the iteration scheme. Once the upper branch is found the first equation can be reduced to a quadratic and the latter two branches obtained in a straightforward manner. This algorithm is quite efficient. as demonstrated by convergence to four significant figures in less than six-seven iterations.

Numerical results are obtained for a variety of cases and are shown in the figures. Figures 1.2 and 3 are amplitude-frequency curves for $i=0,1.2$ respectively. Shown are the present approximation and the usual one term approximation $(k=0)$. Exact results in terms of Jacobian elliptic functions are available for the free vibration case for $\lambda=0.1 .2 .18]$ These are indistinguishable from the present results. Several important features emerge. As $\lambda$ increases, the difference between the two approximations also increases. This is due to the deviation of the $\zeta \tau$ response from the cosine function. e.g., see the $\measuredangle \tau$ inset plots on Figs, 1.2 and 3. This effect is maximum in the forced. low frequency, $\omega<\downarrow p$. response region where many approximation schemes have difficulty. see [12]. Note. for example. the smooth behavior of the cosine approximation on Figs. 1. 2 and 3. The correct response. however, contains strong higher harmonic components [10] which cause the "bump" in the curve at low frequencies. It should be pointed out that the present approximation is also in doubt for $\omega<0.4 \sqrt{ } p$. This is due to the limited two parameter $(.4 . k)$ approximation assumed, i.e.. all harmonies are represented but are not linearly independent. This region can be extended by considering a more general approximation. see equation (2). 


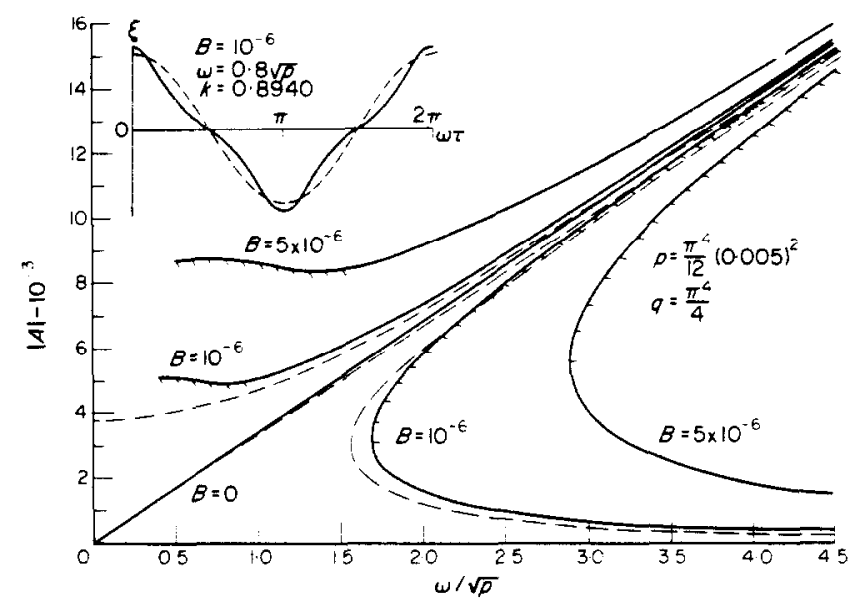

Fig. 2. Frequency-response curves for $j=1$.

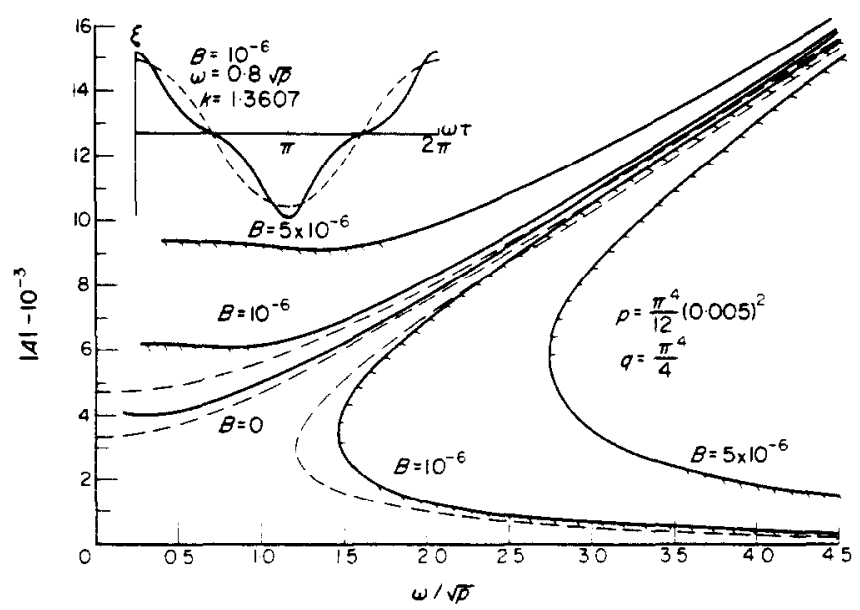

Fig. 3. Frequency-response curves for $i=2$.

Important information concerning the non-linear behavior and the changing form of the $\xi . \tau$ response can be deduced directly from $k$. Figures 4.5 and 6 are plots of $k$ vs forcing frequency for $\lambda=0.1$ and 2 respectively. As (') increases. $k$ tends asymptotically to the free vibration $k$ values (for the p.q parameters chosen) for all $i$ and $B$. indicating little change in form at high frequencies. Note. also. that $k$ becomes large for low frequencies indicating the strong effect of the higher harmonics.

Accuracy of the method is considered in two ways. Where exact results exist, as in certain cases of free vibration, the approximation is identical to the exact results to the fourth significant figure for the entire range considered. $0 \leqslant(1) \leqslant 4.5, \mathrm{p} .0 \leqslant i \leqslant 2$. Note that the system considered is strongly non-linear. + For the forced response (no exact

t The numerical values of p.q. B. i used in the computations are listed on the figures. 


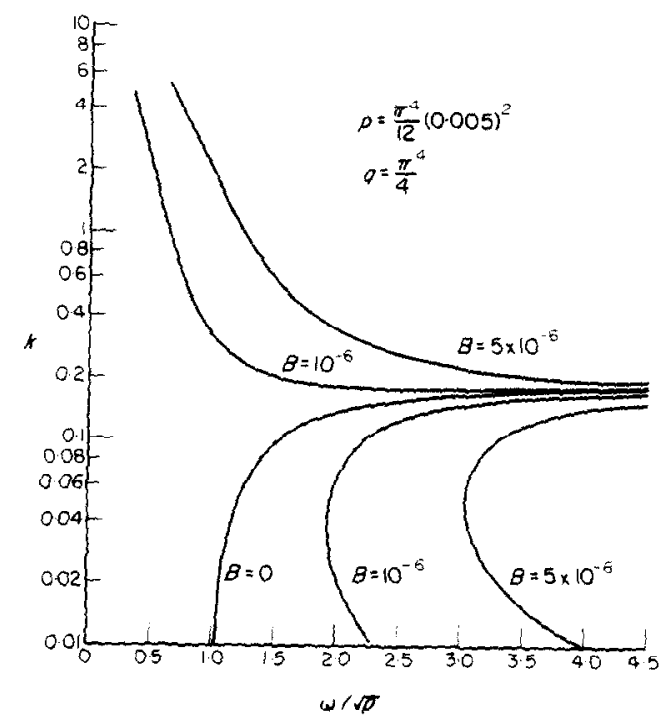

Fig. 4. Form parameter $k$ vs frequency $(\omega, p$ for $\lambda=0$.

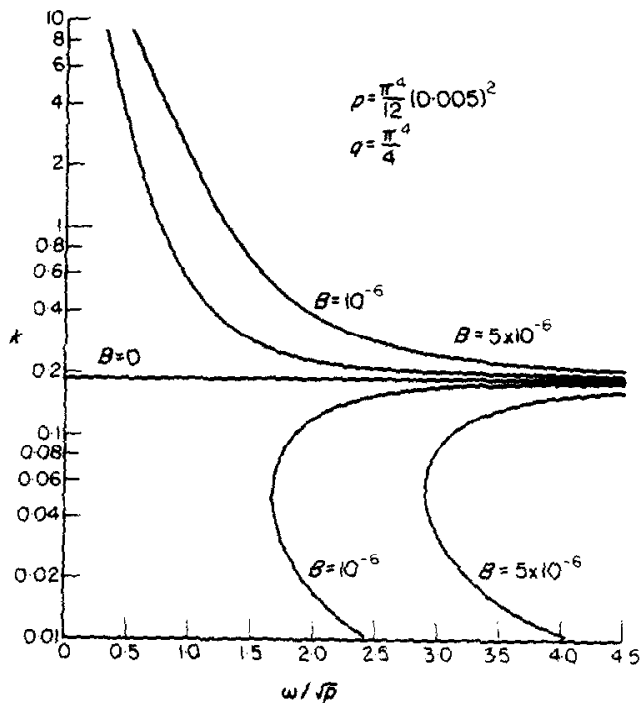

Fig. 5. Form parameter $k$ vs frequency $(2, p$ for $\dot{i}=1$.

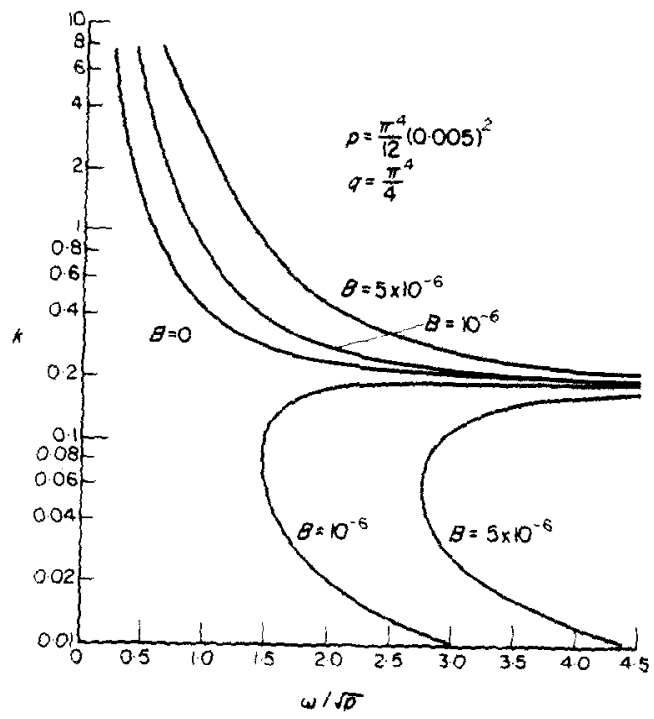

Fig. 6. Form parameter $k$ ws irequency 1 \% $v p$ for $i=2$.

results available), comparison of the present approximation is made with the cosine approximation. The square of the residual is summed over one cycle, equation (5), for several representative points in the amplitude frequency spectrum for both approximations and the results presented in the table below. Note that the present approximation is in general two orders of magnitude better. 
Table 1. Comparison of form function. cosine. approximations

\begin{tabular}{|c|c|c|c|c|c|c|c|c|}
\hline \multirow{2}{*}{ i. } & \multirow{2}{*}{$\frac{a}{19}$} & \multirow{2}{*}{$\begin{array}{c}\text { Solution } \\
\text { branch }\end{array}$} & \multicolumn{2}{|c|}{$B=0$} & \multicolumn{2}{|c|}{$B=10^{-n}$} & \multicolumn{2}{|c|}{$B=5 \times 10^{\cdots}$} \\
\hline & & & FFA & $\mathrm{CA}$ & FFA & $\mathrm{CA}$ & FFA & $\mathrm{CA}$ \\
\hline \multirow{2}{*}{0} & 0.8 & $\begin{array}{l}1 \\
3\end{array}$ & $\begin{array}{l}\text { N.S. } \\
\text { N.S. }\end{array}$ & $\begin{array}{l}\text { N.S. } \\
\text { N.S. }\end{array}$ & $\begin{array}{c}1.7 \times 10^{-13} \\
\text { N.S. }\end{array}$ & $\begin{array}{c}1.7 \times 10^{-11} \\
\text { N.S. }\end{array}$ & $\begin{array}{c}4.6 \times 10^{-10} \\
\text { N.S. }\end{array}$ & $\begin{array}{c}6.3 \times 10^{-10} \\
\text { N.S. }\end{array}$ \\
\hline & $3 \cdot 0$ & $\begin{array}{l}1 \\
3\end{array}$ & $\begin{array}{c}58 \times 10^{-12} \\
\text { N.S. }\end{array}$ & $\begin{array}{c}2.0 \times 10^{-9} \\
\text { N.S. }\end{array}$ & $\begin{array}{l}8.0 \times 10^{-12} \\
2.0 \times 10^{-12}\end{array}$ & $\begin{array}{l}2.4 \times 10^{-4} \\
2.4 \times 10^{-9}\end{array}$ & $\begin{array}{c}2.9 \times 10^{-11} \\
\text { N.S. }\end{array}$ & $\begin{array}{c}5.3 \times 10^{-9} \\
\text { N.S. }\end{array}$ \\
\hline \multirow{2}{*}{1} & 0.8 & $\begin{array}{l}1 \\
3\end{array}$ & $\begin{array}{c}1.4 \times 10^{-14} \\
\text { N.S. }\end{array}$ & $\begin{array}{c}3.6 \times 10^{-12} \\
\text { N.S. }\end{array}$ & $\begin{array}{c}1.8 \times 10^{-12} \\
\text { N.S. }\end{array}$ & $\begin{array}{c}7.6 \times 10^{-11} \\
\text { N.S }\end{array}$ & $\begin{array}{c}5.4 \times 10^{-10} \\
\text { N.S. }\end{array}$ & $\begin{array}{c}1.1 \times 10^{-9} \\
\text { N.S. }\end{array}$ \\
\hline & 30 & $\begin{array}{l}1 \\
3\end{array}$ & $\begin{array}{c}1.1 \times 10^{-11} \\
\text { N.S. }\end{array}$ & $\begin{array}{c}2 \cdot 8 \times 10^{-4} \\
\text { N.S. }\end{array}$ & $\begin{array}{l}1.4 \times 10^{-11} \\
2.6 \times 10^{-12}\end{array}$ & $\begin{array}{l}3.3 \times 10^{-9} \\
3.3 \times 10^{-9}\end{array}$ & $\begin{array}{l}3.2 \times 10^{-11} \\
1.1 \times 10^{-10}\end{array}$ & $\begin{array}{l}5.4 \times 10^{-9} \\
54 \times 10^{-9}\end{array}$ \\
\hline \multirow{2}{*}{2} & 0.8 & $\begin{array}{l}1 \\
3\end{array}$ & $\begin{array}{c}1.4 \times 10^{-12} \\
\text { N.S. }\end{array}$ & $\begin{array}{c}6.2 \times 10^{-11} \\
\text { N.S. }\end{array}$ & $\begin{array}{c}9.3 \times 10^{-12} \\
\text { N.S. }\end{array}$ & $\begin{array}{c}2.4 \times 10^{-10} \\
\text { N.S. }\end{array}$ & $\begin{array}{c}6.3 \times 10^{-10} \\
\text { N.S. }\end{array}$ & $\begin{array}{c}1.7 \times 10^{-9} \\
\text { N.S }\end{array}$ \\
\hline & 3.0 & $\begin{array}{l}1 \\
3\end{array}$ & $\begin{array}{c}1.8 \times 10^{-11} \\
\text { N.S. }\end{array}$ & $\begin{array}{c}3.8 \times 10^{-4} \\
\text { N.S. }\end{array}$ & $\begin{array}{l}2.2 \times 10^{-11} \\
3.7 \times 10^{-12}\end{array}$ & $\begin{array}{l}4.4 \times 10^{-4} \\
4.4 \times 10^{-4}\end{array}$ & $\begin{array}{l}4.8 \times 10^{-11} \\
1.3 \times 10^{-10}\end{array}$ & $\begin{array}{l}6.8 \times 10^{-9} \\
6.8 \times 10^{-9}\end{array}$ \\
\hline
\end{tabular}

Note: FFA is the form function approximation. CA is the cosine approximation: N.S. is no solution. Solution branches are numbered with decreasing amplitude.

\section{STABILITY}

The assumed steady-state solution. equation (20). represents a periodic state of equilibrium. The physical existence of this solution depends on its stability; which will now be considered in the classical manner: we let

$$
\sqsubseteq(\tau)=\xi_{s s}(\tau)+\eta(\tau)
$$

where $\eta(\tau)$ is a small perturbation of the assumed steady-state solution $\xi_{s s}(\tau)$. Substituting equation (26) into equation (1) and neglecting higher-order terms of $\eta(\tau)$. we obtain the following variational equation for $\eta(\tau)$

$$
\frac{\mathrm{d}^{2} \eta}{\mathrm{d} \tau^{2}}+\left[p(1-\lambda)+3 q A^{2} \mathrm{e}^{-2 k \sin ^{2} \omega \tau} \cos ^{2}(1)\right] \eta(\tau)=0
$$

Since the coefficient of the $\eta(\tau)$ term is periodic. equation (27) is a form of Hill's equation. If the solution. $\eta(\tau)$. is bounded. the corresponding steady-state solution $\xi_{s s}(\tau)$. is stable.

Equation (27) can be put into a standard form. see [6]. by introducing the change of variable $\tau=\omega \tau$ and expanding the periodic coefficient into a Fourier series in the interval $0 \leqslant \tau \leqslant 2 \pi$. i.e.

$$
\frac{\mathrm{d}^{2} \eta}{\mathrm{d} \tau}+\left[\theta_{0}+2 \sum_{n=1}^{\infty} \theta_{n} \cos 2 n \tau\right] \eta(\tau)=0
$$


where

$$
\begin{aligned}
& \theta_{0}=\frac{p(1-i)}{\omega^{2}}+\frac{3}{2} \frac{q}{\omega^{2}} A^{2}\left[z_{0}(k)+z_{1}(k)\right] \\
& \theta_{1}=\frac{3}{2} \frac{q}{\omega^{2}} A^{2}\left[z_{0}(k)+\left(1-\frac{1}{k}\right) z_{1}(k)\right] \\
& \theta_{2}=\frac{3}{2} \frac{q}{\omega^{2}} A^{2}\left[\left(1-\frac{2}{k}\right) z_{0}(k)+\left(1-\frac{2}{k}+\frac{4}{k^{2}}\right) z_{1}(k)\right] \\
& \theta_{3}=\frac{3}{2} \frac{q}{\omega^{2}} A^{2}\left[\left(1-\frac{4}{k}+\frac{12}{k^{2}}\right) z_{0}(k)+\left(1-\frac{5}{k}+\frac{8}{k^{2}}-\frac{24}{k^{3}}\right) z_{1}(k)\right] \\
& \theta_{4}=\frac{3}{2} \frac{q}{\omega^{2}} A^{2}\left[\left(1-\frac{8}{k}+\frac{24}{k^{2}}-\frac{96}{k^{3}}\right) z_{0}(k)+\left(1-\frac{8}{k}+\frac{40}{k^{2}}-\frac{48}{k^{3}}+\frac{192}{k^{4}}\right) z_{1}(k)\right]
\end{aligned}
$$

In the cases under consideration here, the series

$$
\sum_{n=1}^{x}\left|\theta_{n}\right|
$$

converges rapidly, and it is sufficient to retain the first four terms.

By the use of Floquet's theory and a method similar to Whittaker's method for solving Mathieu's equation. see Ince.[13] higher-order solutions of equation (28) in the first three unstable regions are calculated. These are tabulated in Appendix II of Hayashi, [6] and the results can be used to establish the values of $\theta_{0}$ on the first three sets of boundaries separating the regions of bounded and unbounded solutions.

$$
\begin{aligned}
& ()_{o l}^{(1)} \cdot \theta_{o r}^{(1)}=1 \mp \theta_{1}-\frac{1}{8}\left(\theta_{1}^{2}-\frac{1}{6}()_{2}^{2}-\frac{1}{16} \theta_{3}^{2} \mp \frac{1}{4} \theta_{1}\left(\theta_{2}\right.\right. \\
& \mp \frac{1}{12} \theta_{2} \theta_{3} \pm \frac{1}{64} \theta_{1}^{3}+\frac{1}{48} \theta_{1}^{2} \theta_{2} \mp \frac{5}{192} \theta_{1}^{2} \theta_{3} \pm \frac{1}{144} \theta_{1} \theta_{2}^{2} \pm \frac{1}{2304} \theta_{1} \theta_{3}^{2} \\
& \mp \frac{1}{48} \theta_{2}^{2} \theta_{3}-\frac{13}{288} \theta_{1} \theta_{2} \theta_{3}+\ldots \\
& \left(\theta _ { o l } ^ { ( 2 ) } \cdot \left(\theta_{o r}^{(2)}=4 \mp \theta_{2}+\left(\frac{1}{6} \pm \frac{1}{4}\right)\left(\theta_{1}^{2}-\frac{1}{32}()_{2}^{2}-\frac{1}{10} \theta\right)_{3}^{2}-\frac{1}{24} \theta \theta_{4}^{2}\right.\right. \\
& \mp \frac{1}{6} \theta_{1} \theta_{3} \mp \frac{1}{16} \theta_{2} \theta_{4} \pm \frac{1}{1024} \theta_{2}^{3}+\left(\frac{19}{192} \pm \frac{1}{9}\right) \theta_{1}^{2} \theta_{2} \mp \frac{7}{576} \theta \theta_{1}^{2} \theta_{4}+\frac{1}{768} \theta \frac{2}{2} \theta_{4} \\
& \pm \frac{1}{225}\left(\theta _ { 2 } \left(\theta_{3}^{2} \pm \frac{1}{2304}\left(\theta_{2}\left(\theta \frac{2}{4} \mp \frac{5}{576} \theta\right)_{3}^{2} \theta_{4}\right.\right.\right. \\
& +\left(\frac{97}{1440} \pm \frac{5}{96}\right) \theta_{1}\left(\theta_{2} \theta_{3}+\left(\frac{1}{360} \pm \frac{11}{480}\right) \theta_{1}\left(\theta_{3} \theta_{4}+\ldots .\right.\right.
\end{aligned}
$$




$$
\begin{aligned}
\theta_{o l}^{(3)}, \theta_{o r}^{(3)}= & 9 \mp \theta_{3}+\frac{1}{16} \theta_{1}^{2}+\frac{1}{10} \theta_{2}^{2}-\frac{1}{72} \theta_{3}^{2}-\frac{1}{14} \theta_{4}^{2} \\
& \pm \frac{1}{4} \theta_{1} \theta_{2} \mp \frac{1}{8} \theta_{1} \theta_{4} \mp \frac{1}{64} \theta_{1}^{3} \pm \frac{1}{5184} \theta_{3}^{3} \\
& -\frac{3}{160} \theta_{1}^{2} \theta_{2} \pm \frac{9}{256} \theta_{1}^{2} \theta_{3} \pm \frac{9}{400} \theta_{2}^{2} \theta_{3}+\frac{3}{140} \theta_{2}^{2} \theta_{4} \\
\pm & \frac{9}{3136} \theta_{3} \theta_{4}^{2}+\frac{73}{1440} \theta_{1} \theta_{2} \theta_{3} \pm \frac{3}{160} \theta_{1} \theta_{2} \theta_{4} \\
& \quad+\frac{103}{4032} \theta_{1} \theta_{3} \theta_{4} \pm \frac{5}{288} \theta_{2} \theta_{3} \theta_{4}+\ldots
\end{aligned}
$$

where the numerical superscripts represent the orders of the unstable regions. and the subscripts $l$ and $r$ stand for their left and right boundaries respectively. In addition we have

$$
\theta_{o r}^{(i)} \leqslant \theta_{o i}^{(i+1)} \quad i=1,2 \ldots
$$

where this condition does not hold. $\theta_{o l}^{(i-1)}$ assumes the value $\theta_{a r}^{(i)}$.

Note that there exists two simplified stability analyses for the Hill equation. In the first. higher order terms of $\theta_{n}$ in equation (30) are neglected. This leads to linear stability boundaries in the $\theta_{0} . \theta_{n}$ plane. These results can lead to erroneous stability predictions in certain regions of the amplitude frequency plot. In the second case. $\theta_{1}$ is much larger numerically than $\theta_{n}, n>1$. The Hill equation simply reduces to a form of Mathieu's equation. the stability properties of which are well known.

In the present study, however. neither approximation provides sufficient accuracy, An algorithm based on the full form of equation $(30)$ is used. The values of $\theta_{0}$ and $\theta_{n}$ 's are obtained for a given point in the frequency-amplitude space. The calculated value of $\theta_{0}$ (response point) is then compared with $\theta_{o l}^{i j} . \theta_{o r}^{(i)}$ and is in the $i^{\text {th }}$ unstable region if

$$
\theta_{o l}^{(i)}<\theta_{0}<\theta_{o r}^{(i)} \quad i=1.2 \ldots
$$

except for the low frequency range consideration of the first three unstable regions. $i=1.2 .3$, is sufficient. Results of the stability investigation are shown on the amplitude frequency plots. Figs. 1-3, i.e. unstable response points are flagged.

\section{CONCLUSIONS AND DISCLSSION}

A new type of approximating function has been used to determine the steady-state response and stability of the forced Duffing equation. This function has two important features. It is capable of exhibiting large curvatures and due to the non-linear appearance of the unknown parameter the "form" can change throughout the non-linear range. Thus single term (two parameter) solutions provide very accurate results throughout the non-linear range. In terms of numerical effort. a comparison with the harmonic balance method, for example. indicates that for equivalent accuracy the form function approach requires less effort. This is due to the presence of all higher harmonic components in the form function approximation.

The form function approximation has currently been used in the solution of the above mentioned problem and the large deflection of plates. [14] It is felt. however, that there are many mechanics problems for which the method would be useful. namely those in which the dependent variables undergo large and changing curvatures in the non-linear range. 
There are several areas of future investigation. Additional rationale is necessary for narrowing the selection process of the $G_{i}$ s. This is being investigated through the use of asymptotic methods. Also. it should be pointed out that for the current class of problems investigated the location of maximum curvature are known. For many physical problems of interest this is the case. When indeterminate. however, it is not clear at present how to choose the form function. A possible approach is to consider the position as yet another unknown parameter. The space and time problems mentioned above can also be combined to solve the complete problem of the non-linear vibration of buckled beams plates. This problem is currently under investigation.

\title{
REFERENCES
}

1. B. A. Finlayson and L. E. Scriven. The methods of weighted residuats - a review. tppl. Mech. Ret. 19.735.748 $(1966)$.

2. J. G. Elsey. Non-linear vibration of beams and rectangular plates. J. Appl. Wath. Phys. 15. 167-175(1964).

3. J. A. Bennett and J. G. Eisley. A multiple degree-of-freedom approach to nun-tintear beam vibrations. AlA.A J.8.734-739(1970).

4. W. Y. Tseng and J. Dugundji. Non-linear vibrations of a buckled beam under harmonic excitation. $J$. 4ppl. Hech. 38, 467-476 (1971).

5. K. O. Friedrichs and J. J. Stoker. Fored vibrations of systems with non-linear restoring force. Q. Appl. Math. 1. No. 2.97-11511943\}.

6. C. Hayashi. Non-linear Oscillations in Phrsical Sistems. McGraw-Hill. New York (1964).

7. W. J. Cunningham. Introduction to Von-lincar thalysis. McGraw-Hill. New York (1958).

8. D. Burgreen. Free vibrations of a pin-ended column with constant distance between pin ends. J. Appl. Mech. 18. $135-139(1951)$.

9. W. D. Iwan and E. J. Patula. The merit of different error minimization criteria in approximate analysis. J. Appl. Mech. 39.257.26211972)

10. D. Young. Forced vibration of system with non-linear restoring force. Proc. Ist midwestern Conf. solid . Mech. Urbana. Ill.. 164-169 (1953).

11. B. Carnaham. H. A. Luther. and J. O. Wilkes. Applied Vumerical Methods, John Wiley. New York (1969).

12. C. R. Wylie. Jr.. On the forced vibrations of non-linear springs. J. Franklin Inst. 236. 273-284 (1943).

13. E. L. Ince. On a general solution of Hill's equation, Monthly notices $R$. Astron. Soc. 75, 436 448 (1915): 76. $431-442(1916): 78$. $141-147(1917)$.

14. D. L. Sikarskie and C. L. Lou. On the improsenent of itpproximate solution for large dellections of plates. Proc: loth Structures. Structural Dinamies and Materials Conf. New Orleans, 151-157 1969).

\begin{abstract}
Résumé--On considere un nouveau type de solution dessai qui différe de la combinaison linéaire habituclle de fonctions d'approximation. Il nécessite de modifier les fonctions d'approximation avec des "fonctions de forme": fonctions contenant des parametres indetermines apparaissant non lineairement. un hon choix de ees fonctions fournit une approximation plus fine des importantes courbures locales qui apparaissent dans certains problemes non lineaires. Dans cet article on montre lapproximation de la "fonction de forme" pour des solutions en regime permanent de léquation de Duffing. Cette equation apparait dans le probleme de vibrations non linéaires de poutres et de plaques apres tambage. On montre que le comportement en stabilité da ces solutions en régime permanent ust régi par une équation de Hill. On trouve que lapproximation de la "fonction de forme" donne des resultats numertques notablement meilleurs que. par exemple. ceux donnes par la méthode de balance harmonique. La méthode fournit egalement des indications supplémentaires sur le comportement non lineadire. particulièrement dans la region des reponses a bisse frequence.
\end{abstract}

Zusammenfassung - Eıne neue Art von Versuchsiösungen wird untersucht. diesich von den übtichen Linearkombinationen von Annäherungstunktionen unterscheiden. Das Verfahren beinhaltet die Abänderung der Anniherungsfunktionen durch "Formfunktinen", die nichtlinear auftretende unhestimmte Parameter enthalten. Die richtige Wahl dieser Parameter ergiht eine hessere Annäherung für die grossen örtichen Krümmungen, die in einigen nichtlinearen Problemen 
auftreten. In dieser Arbeit wird die Annäherung durch "Formfunktionen" für die stationören Lösungen der Duffing-Gleichung demonstriert. Diese Gleichung tritt bei nichtlinearen Schwingungen ausgeknickter Träger und Platten auf. Es wird gezeigt. dass das Stabilitätsverhalten dieser stationären Lösungen durch die Hillsche Gleichung beschrieben wird. Es zeigt sich. dass die Annäherung durch "Formfunktionen" merklich bessere numerische Ergbnisse liefert als die z.B. mit der Methode des harmonischen Ausgleichs erhaltenen. Die Methode gestattet auch ein besseres Verständnis von nichtlinearem Verhalten. besonders im Gebiet niedriger Frequenzen.

Аннотация - Рассматриваются приближенные решения нового типа, отличаюшиеся от обычно употребляемых линейных комбинаций аппроксимируюших функщий. В новом методе аппроксимируюшие функции модифишируются при помоши «функций формы»; функции, содержащие неопределенные параметры входят нелинейно, и соответствуюший их выбор обеспечивает более точную аппроксимацию при больших локальных кривизнах, которые возникают в некоторых нелинейных задачах. В данной работе аппроксимашия «функииями формы» демонстрируется на примере стационарного решения уравнения Дуффинга, которое описывает нелинейные колебания балок и пластинок при потере устойчивости. Показано, что устойчивое поведение этих стационарных решений описывается уравнением Хилла. Обнаружено, что аппроксимация «функциями формы» приводит к значительно пучшим численным результатам, чем, например, результаты, получаемые по методу гармонического баланса. Данный метод дает также дополнительные сведения о нелинейном поведении, в частности, в области низких частот. 